\title{
UNIFORM CONVEXITY. III
}

\author{
MAHLON M. DAY
}

It is the purpose of this note to fill out certain results given in two recent papers on uniform convexity of normed vector spaces. ${ }^{1}$ A normed vector space ${ }^{2} B$ is called uniformly convex with modulus of convexity $\delta$ if for each $\epsilon>0$ there exists a $\delta(\epsilon)>0$ such that for every two points $b$ and $b^{\prime}$ of $B$ satisfying the conditions $\|b\|=\left\|b^{\prime}\right\|=1$ and $\left\|b-b^{\prime}\right\| \geqq \epsilon$ the quantity $\left\|b+b^{\prime}\right\| \leqq 2(1-\delta(\epsilon))$. If $\left\|b_{0}\right\|=1, B$ is said to be locally uniformly convex near $b_{0}$ if there is a sphere about $b_{0}$ in which the condition for uniform convexity holds. Theorem 1 shows that all properties of normed vector spaces which are invariant under isomorphism are the same for uniformly convex and locally uniformly convex spaces. Theorem 2 gives a necessary condition for isomorphism with a uniformly convex space. The condition is in terms of isomorphisms of finite dimensional subspaces and is suggested by examples given in [I]; it is not known whether the condition is sufficient. Theorem 3 is somewhat more general than Theorem 3 of [II]; it uses uniformly convex function spaces instead of the $l_{p}$ spaces of [II].

A cone $C$ in $B$ is a set which contains all of every half line from the origin through each point of $C$.

LEMMA 1. A normed vector space $B$ is locally uniformly convex near $b_{0}$ if and only if there exists a convex cone $C$, with $b_{0}$ in its interior, such that for every $\epsilon$ there is a $\delta_{1}(\epsilon)>0$ such that the conditions $\|b\| \leqq 1$, $\left\|b^{\prime}\right\| \leqq 1$, and $\left\|b-b^{\prime}\right\| \geqq \epsilon$ imply $\left\|b+b^{\prime}\right\| \leqq 2\left(1-\delta_{1}(\epsilon)\right)$ for every pair of points $b$ and $b^{\prime}$ in $C$.

If this condition is satisfied there is obviously a sphere about $b_{0}$ inside $C$, so that in that sphere $\delta(\epsilon)$ can be taken equal to $\delta_{1}(\epsilon)$. On the other hand, if there is a sphere of radius $2 k$ about $b_{0}$ in which $\delta$ can be defined, it can be shown that it suffices to let $C$ be the cone through points of the sphere of radius $k$ about $b_{0}$ and to let $\delta_{1}(\epsilon)=\inf [\epsilon / 10, \delta(4 \epsilon / 5) / 2]$.

LEMma 2. If the cone $C$ of Lemma 1 contains a sphere about $b_{0}$ of radius $k$, if $\|b\| \leqq 1$ and if $\left\|b-b_{0}\right\| \geqq k$, then $\left\|b+b_{0}\right\|<2-\delta_{1}(k)$.

Presented to the Society, April 24, 1943; received by the editors January 25, 1943.

1 These papers are [I] Reflexive Banach spaces not isomorphic to uniformly convex spaces, Bull. Amer. Math. Soc. vol. 47 (1941) pp. 313-317, and [II] Some more uniformly convex spaces, Bull. Amer. Math. Soc. vol. 47 (1941) pp. 504-507.

${ }^{2}$ See Banach, Thêorie des opérations linéaires, Warsaw, 1932, for general definitions. 
This is obvious if $\left\|b-b_{0}\right\|=k$. If $\|b\| \leqq 1$ and $\left\|b-b_{0}\right\|>k$ there exists a point $b_{1}=\lambda b+(1-\lambda) b_{0}, 0<\lambda<1$, on the line segment from $b_{0}$ to $b$ such that $\left\|b_{0}-b_{1}\right\|=k$ while $\left\|b_{1}\right\| \leqq 1$; hence $\left\|b_{1}+b_{0}\right\| \leqq 2\left(1-\delta_{1}(k)\right)$. Let $f$ be a linear functional such that $f\left(b^{\prime}\right) \leqq\left\|b^{\prime}\right\|$ for all $b^{\prime}$ in $B$ and such that the line $\left\{b^{\prime} \mid f\left(b^{\prime}\right)=1\right\}$ in the plane of $0, b_{0}$ and $b$ touches the unit sphere in $B$ at the point of intersection of that sphere with the half line from 0 through $b+b_{0}$, so that $f\left(b+b_{0}\right)=\left\|b+b_{0}\right\|$. Then $f\left(b_{0}+b_{1}\right) \leqq\left\|b_{0}+b_{1}\right\| \leqq 2\left(1-\delta_{1}(k)\right)$. Two cases can now be distinguished: If $f\left(b_{0}\right) \geqq f(b), 2-2 \delta_{1}(k) \geqq f\left(b_{1}+b_{0}\right) \geqq f\left(b+b_{0}\right)=\left\|b+b_{0}\right\|$. If $f\left(b_{0}\right)<f(b)$, $2\left(1-\delta_{1}(k)\right) \geqq f\left(b_{1}+b_{0}\right)=f\left(b_{1}\right)+f\left(b_{0}\right)>2 f\left(b_{0}\right)$, so $\left\|b+b_{0}\right\|=f\left(b+b_{0}\right)=f\left(b_{0}\right)$ $+f(b)<1-\delta_{1}(k)+1$.

THEOREM 1. If $B$ is locally uniformly convex near some point $b_{0}$, then $B$ is isomorphic to a uniformly convex space. If $k$ is the radius of the sphere which exists by Lemma 1 about $b_{0}$, a suitable modulus of convexity for the new space is given in terms of the old by $\delta_{1}^{\prime}(\epsilon)=1$ $-1 /\left[1+\delta_{1}\left(\delta_{1}(k) \epsilon / 4\right) /\left(k+\delta_{1}(k) / 4\right)\right]$.

Suppose the cone $C$ of Lemma 1 contains a sphere $\left\{b \mid\left\|b-b_{0}\right\| \leqq k\right\}$ about $b_{0}$; let $\alpha=1-\delta_{1}(k) / 4$ and consider the two spheres $E_{1}=\left\{b \mid\left\|b-\alpha b_{0}\right\| \leqq 1\right\}$ and $E_{2}=\left\{b \mid\left\|b+\alpha b_{0}\right\| \leqq 1\right\}$. If $S$ is the intersection of $E_{1}$ and $E_{2}$, it is clear that $S$ is convex and symmetric about the origin, and that $S$ contains the sphere $\left\{b \mid\|b\| \leqq \delta_{1}(k) / 4\right\}$.

To show $\|b\| \leqq k+\delta_{1}(k) / 4$ for each $b$ in $S$, it suffices to show that $b \in S$ implies that $b+\alpha b_{0}$ is within $k$ of $b_{0}$. If this is false, that is, if $\left\|b+\alpha b_{0}-b_{0}\right\|>k$, then, by Lemma $2,\left\|b+\alpha b_{0}+b_{0}\right\|<2-\delta_{1}(k)$. How ever $\left\|b+\alpha b_{0}+b_{0}\right\|=\left\|b-\alpha b_{0}+(1+2 \alpha) b_{0}\right\| \geqq(1+2 \alpha)\left\|b_{0}\right\|-\left\|b-\alpha b_{0}\right\| \geqq 1$ $+2 \alpha-1=2 \alpha=2-\delta_{1}(k) / 2>2-\delta_{1}(k)$.

Let $|b|$ be the smallest non-negative value of $t$ for which the point $b / t$ is in $S$. Then $|\cdots|$ defines a new norm in $B$ and it is clear from the inequalities thus far derived that $\left[\delta_{1}(k) / 4\right]|b| \leqq\|b\|$ $\leqq\left[k+\delta_{1}(k) / 4\right]|b|$, so this new norm defines a space isomorphic to the original and all that need be proved is that $|\ldots|$ is uniformly convex. If $b_{1}, b_{2} \in S$, and $\left|b_{1}-b_{2}\right|>\epsilon$, then $\left\|\left(b_{1}+\alpha b_{0}\right)-\left(b_{2}+\alpha b_{0}\right)\right\|$ $=\left\|b_{1}-b_{2}\right\| \geqq \delta_{1}(k) \epsilon / 4$. Also $\left\|b_{i}+\alpha b_{0}-b_{0}\right\| \leqq k$ by the preceding paragraph so, by the original hypotheses near $b_{0},\left\|b_{1}+b_{2}+2 \alpha b_{0}\right\|$ $\leqq 2\left[1-\delta_{1}\left(\delta_{1}(k) \epsilon / 4\right)\right] \equiv 2(1-\mu(\epsilon)) ;$ that is, $\left(b_{1}+b_{2}\right) / 2=E_{1}^{\prime}=\left\{b \mid\left\|b+\alpha b_{0}\right\|\right.$ $\leqq 1-\mu(\epsilon)\}$. The same argument with $-\alpha b_{0}$ and $-b_{0}$ shows that $\left(b_{1}+b_{2}\right) / 2 \in E_{2}^{\prime}=\left\{b \mid\left\|b-\alpha b_{0}\right\| \leqq 1-\mu(\epsilon)\right\}$.

It will now suffice to show that there is a $\delta_{1}^{\prime}(\epsilon)>0$ such that $|b|<1-\delta_{1}^{\prime}(\epsilon)$ if $b \in E_{1}^{\prime} \cdot E_{2}^{\prime} . E_{i}^{\prime} \subset E_{i}, i=1,2$, so for any $b$ in $E_{1}^{\prime} \cdot E_{2}^{\prime}$ there is a number $t \geqq 1$ such that $|t b|=1$; hence, either $\left\|t b+\alpha b_{0}\right\|=1$ or $\left\|t b-\alpha b_{0}\right\|=1$. These cases are interchanged by replacing $b$ by 
$-b$ so it suffices to consider the first; then $1-\mu(\epsilon) \geqq\left\|\alpha b_{0}-b\right\|$ $=\left\|\alpha b_{0}-t b+t b-b\right\| \geqq\left\|\alpha b_{0}-t b\right\|-\|t b-b\|=1-(t-1)\|b\|$. Therefore $(t-1)\|b\| \geqq \mu(\epsilon)$ or $t \geqq 1+\mu(\epsilon) /\|b\| \geqq 1+\delta_{1}\left(\delta_{1}(k) \epsilon / 4\right) /\left[k+\delta_{1}(k) / 4\right]$. Letting $1-\delta_{1}^{\prime}(\epsilon)$ be the reciprocal of the last term in the preceding in. equality gives $|b|=1 / t \leqq 1-\delta_{1}^{\prime}(\epsilon)$ if $b \in E_{1}^{\prime} \cdot E_{2}^{\prime}$.

We turn now to a necessary condition for isomorphism of $B$ with a uniformly convex space. The effect of uniform convexity on the finite dimensional subspaces of an isomorphic space was used implicitly in [I]; it is given explicit formulation here. Let $B_{0}$ and $B$ be two normed vector spaces; then there exist linear operations of norm $\leqq 1$ defined on $B_{0}$ with values in $B$. For each such operator $U$ there is a largest number $k_{U}, 0 \leqq k_{U} \leqq 1$, such that $\left\|b_{0}\right\| \geqq\left\|U\left(b_{0}\right)\right\|$ $\geqq k_{U}\left\|b_{0}\right\|$ for each $b_{0}$ in $B_{0}$, and this number $k_{U}$ can be taken as a measure of the distortion of $B_{0}$ under the mapping $U$ into $B$. De. fine $k\left(B_{0}, B\right)$ to be the least upper bound of $k_{U}$ as $U$ runs over the linear operators from $B_{0}$ to $B$ of norm not greater than 1; explicitly, $k\left(B_{0}, B\right)=\sup _{\|U\| \leqq} \inf _{\left\|b_{0}\right\|=1}\left\|U\left(b_{0}\right)\right\| . k\left(B_{0}, B\right)$ is then a measure of how nearly $B_{0}$ approaches isometry with a subspace of $B$; if $k\left(B_{0}, B\right)=1$, there are operations which come arbitrarily near preserving distances; $k\left(B_{0}, B\right)>0$ if and only if $B_{0}$ is isomorphic to a subspace of $B$. For the present it suffices to choose certain finite dimensional spaces for $B_{0}$. Let $M_{n}$ and $L_{n}$ be the $n$-dimensional spaces of sequences $t=\left(t_{1}, \cdots, t_{n}\right)$ of $n$ real numbers, where

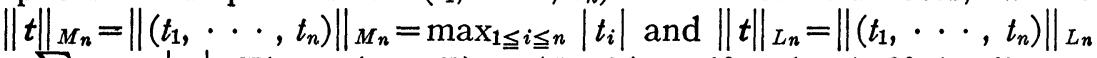
$=\sum_{1 \leqq i \leqq n}\left|t_{i}\right|$. Then $k\left(M_{n}, B\right)=k\left(L_{n}, B\right)=0$ if and only if the dimension of $B$ is less than $n$; also $k\left(M_{n}, B\right)$ and $k\left(L_{n}, B\right)$ are nonincreasing functions of $n$ for each $B$.

LemMa 3. If $U$ is a one-to-one linear operator from $B_{1}$ onto $B_{2}$ such that for some $a \geqq 0,\left\|b_{1}\right\| \geqq\left\|U b_{1}\right\| \geqq a\left\|b_{1}\right\|$ for each $b_{1}$ in $B_{1}$, then for any normed vector space $T, k\left(T, B_{1}\right) \geqq a k\left(T, B_{2}\right) \geqq a^{2} k\left(T, B_{1}\right)$.

If $a=0$, this is obvious. If $a>0$ and $F$ is any linear operator from $T$ into $B$, with $\|F\| \leqq 1$, let $U F$ be defined by $U F(t)=U(F(t))$ for every $t$ in $T$. Then $\|U F\| \leqq 1$ and $\|U F(t)\| \geqq a\|F(t)\|$ for every $t$. Hence $\inf _{\|t\|=1}\|U F(t)\| \geqq a \inf _{\| t||=1}\|F(t)\|$ so $k\left(T, B_{2}\right) \geqq a k\left(T, B_{1}\right)$. If $F^{\prime}$ is any linear operator of norm $\leqq 1$ from $T$ into $B_{2}$, the same argument, using the operator $a U^{-1} F^{\prime}$, shows that $k\left(T, B_{1}\right) \geqq a k\left(T, B_{2}\right)$.

Note that if $U$ maps $B_{1}$ on only part of $B_{2}$ or is not $1-1$ but is of norm $\leqq 1$, the first half of the proof still holds (although $a=0$ in the second case); it follows that if $B_{1}$ is a subspace of $B_{2}$, then $k\left(T, B_{1}\right)$ $\leqq k\left(T, B_{2}\right)$. 
Sobczyk ${ }^{3}$ has defined a special embedding of $l_{1}$ into $m$ which can easily be modified to define an isometry of $L_{n+1}$ and a subspace of $M_{2^{n}}$ so $k\left(L_{n+1}, B\right) \geqq k\left(M_{2^{n}}, B\right)$ for every integer $n$. In particular, $L_{2}$ and $M_{2}$ are isometric so $k\left(L_{2}, B\right)=k\left(M_{2}, B\right)$.

LEMmA 4. If $\delta_{1}$ satisfies Lemma 1 in the whole unit sphere of $B$ and is continuous on the left, then

(1) $k\left(M_{n}, B\right) \leqq\left[1-\delta_{1}\left(2 k\left(M_{n}, B\right)\right)\right]^{n-1}$,

(2) $k\left(L_{2^{n}}, B\right) \leqq\left[1-\delta_{1}\left(2 k\left(L_{2^{n}}, B\right)\right)\right]^{n}$.

If $F$ is an operation from $M_{n}$ into $B$ such that $\|t\| \geqq\|F(t)\| \geqq k\|t\|$ for all $t$, where $k>0$, let $\epsilon_{i}= \pm 1$ for $i=1, \cdots, n$; then the points $F\left(\epsilon_{1}, \cdots, \epsilon_{n}\right)$ lie in the unit sphere of $B$ since $\left\|F\left(\epsilon_{1}, \cdots, \epsilon_{n}\right)\right\|$ $\leqq\left\|\epsilon_{1}, \cdots, \epsilon_{n}\right\|=1$. If $\epsilon_{1}, \cdots, \epsilon_{n}$ and $\epsilon_{1}^{\prime}, \cdots, \epsilon_{n}^{\prime}$ are different, $\left\|F\left(\epsilon_{1}, \cdots, \epsilon_{n}\right)-F\left(\epsilon_{1}^{\prime}, \cdots, \epsilon_{n}^{\prime}\right)\right\| \geqq k\left\|\left(\epsilon_{1}, \cdots, \epsilon_{n}\right)-\left(\epsilon_{1}^{\prime}, \cdots, \epsilon_{n}\right)\right\|$ $=2 k$; hence $\left\|F\left(\epsilon_{1}, \cdots, \epsilon_{n-1}, 0\right)\right\|=\| F\left(\epsilon_{1}, \cdots, \epsilon_{n-1}, 1\right)-F\left(\epsilon_{1}, \cdots\right.$, $\left.\epsilon_{n-1},-1\right) \| / 2 \leqq 1-\delta_{1}(2 k)$; that is, $\left\|F\left(\epsilon_{1}, \cdots, \epsilon_{n-1}, 0\right) /\left[1-\delta_{1}(2 k)\right]\right\|$ $\leqq 1$ for all $\epsilon_{1}, \cdots, \epsilon_{n-1}$. These points are at least $2 k$ apart for different $\epsilon_{i}$, so this process can be applied $n-1$ times to show that $\left\|F(1,0,0, \cdots, 0) /\left[1-\delta_{1}(2 k)\right]^{n-1}\right\| \leqq 1$. Hence $k=k\|1,0,0, \cdots, 0\|$ $\leqq\|F(1,0,0, \cdots, 0)\| \leqq\left[1-\delta_{1}(2 k)\right]^{n-1}$. Taking $k=k\left(M_{n}, B\right)$ or, if that is impossible, taking the limit as $k$ increases toward $k\left(M_{n}, B\right)$ gives (1).

If $F$ maps $L_{2^{n}}$ into $B$ so that $\|t\| \geqq\|F(t)\| \geqq k\|t\|, k>0$, for all $t$, the same sort of argument can be carried through using the points of $L_{2^{n}}$ which have one coordinate equal to one, the others all zero. It leads to the inequality $k=k\left\|\left(2^{-n}, \cdots, 2^{-n}\right)\right\| \leqq\left\|F\left(2^{-n}, \cdots, 2^{-n}\right)\right\|$ $\leqq\left[1-\delta_{1}(2 k)\right]^{n}$ which gives (2).

THEOREM 2. If $B$ is isomorphic to a space which is locally uniformly convex near any point, then $\lim _{n} k\left(M_{n}, B\right)=\lim _{n} k\left(L_{n}, B\right)=0$.

By Theorem $1, B$ is isomorphic to a uniformly convex space $B^{\prime}$. By Lemma $4, k\left(L_{2^{n}}, B^{\prime}\right)<\left[1-\delta_{1}\left(2 k\left(L_{2^{n}}, B^{\prime}\right)\right)\right]^{n}$ for all $n$. If $k\left(L_{2^{n}}, B^{\prime}\right)$ $>k>0$ for all $n$, then $0<k \leqq k\left(L_{2^{n}}, B^{\prime}\right) \leqq\left(1-\delta_{1}(2 k)\right)^{n} \rightarrow 0$ as $n \rightarrow \infty$; this contradiction and the monotony of $k\left(L_{n}, B^{\prime}\right)$ show that $k\left(L_{n}, B^{\prime}\right)$ $\rightarrow 0$. Lemma 3 shows that $k\left(L_{n}, B\right) \rightarrow 0$ also. A similar proof holds for $k\left(M_{n}, B\right)$; this can also be proved by using the remark before Lemma 4 and the fact that $k\left(L_{n}, B\right) \rightarrow 0$.

This theorem has as a corollary the result of $[\mathrm{I}]:$ If $B=\mathbb{P}^{p}\left(B_{i}\right)$, where $B_{i}=l^{p_{i}}$ or $L^{p_{i}}$, and if the numbers $p_{i}$ are not bounded away from

3 A. Sobczyk, Projection of the space $m$ on its subspace $c_{0}$, Bull. Amer. Math. Soc. vol. 47 (1941) pp. 938-947; the construction is given in the proof of Theorem 3. 
1 and $\infty$, then $B$ is not isomorphic to a uniformly convex space.

It is not difficult to give a direct proof of Theorem 2 not using Theorem 1. I have also shown that if $B^{*}$ is uniformly convex, then $k\left(L_{n}, B\right) \rightarrow 0$ (as does $k\left(M_{n}, B\right)$ ); whether this condition is sufficient as well as necessary for isomorphism of $B$ or $B^{*}$ with a uniformly convex space is a question which $\mathrm{I}$ am, so far, unable to answer.

Some remarks may be made about the minimum values, $k\left(L_{n}\right)$ and $k\left(M_{n}\right)$, of $k\left(L_{n}, B\right)$ and $k\left(M_{n}, B\right)$ taken for $n$ fixed and $B$ varying over the spaces of dimension at least $n . k\left(M_{n}, l^{p}\right)=n^{-1 / p}$ if $2 \leqq p<\infty$ and $k\left(L_{n}, l^{p}\right)=n^{-1 / p^{\prime}}$ if $1 \leqq p \leqq 2$ where $1 / p^{\prime}+1 / p=1$. Hence $k\left(L_{n}\right)$ $\leqq k\left(L_{n}, l^{2}\right)=n^{-1 / 2}$ and $k\left(M_{n}\right) \leqq k\left(M_{n}, l^{2}\right)=n^{-1 / 2}$ for all $n$. The plane with a regular hexagon for unit sphere is an example showing that $k\left(L_{2}\right)=k\left(M_{2}\right) \leqq 2 / 3\left(<2^{-1 / 2}\right)$. A tedious computation has shown that $2 / 3$ is precise; that is, that $k\left(L_{2}\right)=k\left(M_{2}\right)=2 / 3$. So far all my attempts to show $k\left(L_{n}\right)$ and $k\left(M_{n}\right) \geqq 1 / n$ have failed for $n>2$.

The rest of this paper is devoted to extending the results of [II]. A normed vector space $T$ of real-valued functions $t=\left\{t_{s}\right\}$ on some set of indices $S$ will be called a proper function space if for every function $t=\left\{t_{s}\right\}$ in $T$ with $0 \leqq t_{s}$ for all $s$ (a) for every real-valued function $\left\{t_{s}^{\prime}\right\}$ with $0 \leqq t_{s}^{\prime} \leqq t_{s}$ for all $s$, the function $\left\{t_{s}^{\prime}\right\} \in T$ and (b) $0 \leqq\left\|\left\{t_{s}^{\prime}\right\}\right\| \leqq\left\|\left\{t_{s}\right\}\right\|$. If $T$ is a proper function space and $B_{s}, s \in S$, are normed vector spaces, let $\mathcal{P}_{T}\left\{B_{s}\right\}$ be the space of functions $\boldsymbol{b}=\left\{b_{s}\right\}$ where $b_{s} \in B_{s}$ and the function $\left\{\left\|b_{s}\right\|\right\} \in T$; in $\mathscr{P}_{T}\left\{B_{s}\right\}$, $\|\boldsymbol{b}\|=\left\|\left\{b_{s}\right\}\right\|=\left\|\left\{\left\|b_{s}\right\|\right\}\right\|$. (In [II] $S$ was countable and only the special product spaces $\mathscr{P}^{p}\left\{B_{s}\right\}=\mathcal{P}_{l^{p}}\left\{B_{s}\right\}$ were used.)

TheOREM 3. If $T$ is a proper function space, then $\mathcal{P}_{T}\left\{B_{s}\right\}$ is uniformly convex if and only if $T$ is uniformly convex and the spaces $B_{s}$ have a common modulus of convexity.

As the proof follows the lines of the proof of Theorem 3 of [II] except at one point it suffices to give the first half of the sufficiency proof; that is, the special case in which $\|b\|=\left\|b^{\prime}\right\|=1,\left\|b-b^{\prime}\right\| \geqq \epsilon$ and $\left\|b_{s}\right\|=\left\|b_{s}^{\prime}\right\|$ for every $s$. Let $\beta_{s}=\left\|b_{s}\right\|$ and $\gamma_{s}=\left\|b_{s}-b_{s}^{\prime}\right\| ;$ then for each $s,\left\|b_{s}+b_{s}^{\prime}\right\| \leqq 2\left(1-\delta\left(\gamma_{s} / \beta_{s}\right)\right) \beta_{s}$ where $\delta$ is a common modulus of convexity for all $B_{8}$. Hence

$$
\left\|\boldsymbol{b}+b^{\prime}\right\|=\left\|\left\{\left\|b_{s}+b_{s}^{\prime}\right\|\right\}\right\|_{T} \leqq 2\left\|\left\{1-\delta\left(\gamma_{s} / \beta_{s}\right) \beta_{s}\right\}\right\|_{T} .
$$

Clearly $\gamma_{s} \leqq 2 \beta_{s}$ for all $s$; let $E$ be the set of all $s$ for which $\gamma_{s} / \beta_{s}$ $>\epsilon / 4$; then in $F$, the complement of $E, \beta_{s} \geqq 4 \gamma_{s} / \epsilon$. If $\left\{t_{s}\right\}$ is any element of $T$, let $t_{s E}=t_{s}$ if $s \in E, t_{s E}=0$ if $s \notin E$; then

$$
1 \geqq\left\|\left\{\beta_{s}\right\}\right\|_{T} \geqq\left\|\left\{\beta_{s F}\right\}\right\| \geqq\left\|\left\{4 \gamma_{s F} / \epsilon\right\}\right\|=(4 / \epsilon)\left\|\left\{\gamma_{s F}\right\}\right\| \text {. }
$$


Hence $\left\|\left\{\gamma_{s F}\right\}\right\| \leqq \epsilon / 4$ and

$$
\left\|\left\{\gamma_{s E}\right\}\right\|=\left\|\left\{\gamma_{s}\right\}-\left\{\gamma_{s F}\right\}\right\| \geqq\left\|\left\{\gamma_{s}\right\}\right\|-\left\|\left\{\gamma_{s F}\right\}\right\| \geqq 3 \epsilon / 4 .
$$

Hence $\left\|\left\{\beta_{s E}\right\}\right\| \geqq\left\|\left\{\gamma_{s E}\right\}\right\| / 2 \geqq 3 \epsilon / 8$.

Now let $t=\left\{\beta_{s F}\right\}, \quad t^{\prime}=\left\{\beta_{s E}\right\}$ and $t^{\prime \prime}=(1-2 \delta(\epsilon / 4)) t^{\prime}$; then $\left\|t+t^{\prime \prime}\right\| \leqq\left\|t+t^{\prime}\right\|=1$ and $\left\|t+t^{\prime}-\left(t+t^{\prime \prime}\right)\right\|=\left\|t^{\prime}-t^{\prime \prime}\right\|=2 \delta(\epsilon / 4)\left\|t^{\prime}\right\|$ $\geqq 3 \delta(\epsilon / 4) \epsilon / 4$. Call this last quantity $\alpha(\epsilon)$; then

$$
\left\|(1-\delta(\epsilon / 4)) t^{\prime}+t\right\|=(1 / 2)\left\|t+t^{\prime}+t+t^{\prime \prime}\right\| \leqq 1-\delta_{1}(\alpha(\epsilon))
$$

where $\delta_{1}$ is the function which exists in $T$ by Lemma 1. By (1) and (2)

$$
\begin{aligned}
\left\|b+b^{\prime}\right\| & \leqq\left\|\left\{\left(1-\delta\left(\gamma_{s} / \beta_{s}\right)\right) \beta_{s E}\right\}+\left\{\beta_{s F}\right\}\right\| \leqq\left\|(1-\delta(\epsilon / 4)) t^{\prime}+t\right\| \\
& \leqq 1-\delta_{1}(\alpha(\epsilon)) \equiv 1-\delta_{2}(\epsilon) .
\end{aligned}
$$

The remainder of the proof is exactly that given in [II] (beginning with line 4 on p. 506); it shows that a suitable value of $\delta_{3}$ in $\mathscr{P}_{T}\left\{B_{8}\right\}$ is given if $\delta_{3}(\epsilon)=\delta_{1}(\eta)$ where $\eta$ is so chosen that $\eta / 2+\delta_{1}(\eta)<\delta_{2}(\epsilon)$. Since $\delta_{3}$ depends only on the moduli of convexity in $T$ and all $B_{s}$, we have the following result, more general than Corollary 1 of [II].

COROLlaRY. If $\{T\}$ is a collection of proper function spaces, if $\{B\}$ is a collection of normed vector spaces, and if all these spaces have a common modulus of convexity, then all the spaces $\mathcal{P}_{T}\left\{B_{8}\right\}$ with $T$ in $\{T\}$ and all $B_{8}$ in $\{B\}$ have a common modulus of convexity.

Some extensions of Theorem 3 may be made; for instance, it is clear that the condition (a) on a proper function space is imposed to make sure that such functions as $\left\{\left\|b_{s}+b_{s}^{\prime}\right\|\right\}$ are in $T$. For example, if $S$ is a space in which a measure is defined and all $B_{s}$ are the same space $B_{0}$, it suffices to take $T=L_{S}^{p}, 1<p<\infty$ and to consider only Bochner measurable functions ${ }^{4}\left\{b_{s}\right\}$ for which $\left\{\left\|b_{s}\right\|\right\} \in T$. In this case all the functions constructed are again in $T$ so the proof can be carried through showing directly that $L^{p}\left(B_{0}\right)$ is uniformly convex if $1<p<\infty$ and $B_{0}$ is uniformly convex. In fact, if the norm in $T$ satisfies (b) and if it is assumed only that every measurable real-valued function dominated by a function in $T$ is again in $T$, the proof can be carried through for the space of Bochner measurable functions from $S$ into $B$ for which $\left\{\left\|b_{s}\right\|\right\} \in T$.

UNIVERSITY OF ILLINOIS

${ }^{4} \mathrm{~S}$. Bochner, Integration von Funktionen, deren Werte die Elemente eines Vektorraumes sind, Fund. Math. vol. 20 (1933) pp. 262-276. 